OPEN ACCESS

Edited by:

Niels Schaft,

University Hospital Erlangen, Germany

Reviewed by:

Hua Zhong,

New York University, United States

German Demidov,

University of Tuebingen, Germany

Michal Lotem,

Hadassah Medical Center, Israel

*Correspondence:

LU Si

silu15_silu@126.com

Jun Guo

guoj307@126.com

${ }^{\dagger}$ These authors have contributed equally to this work

Specialty section: This article was submitted to

Cancer Immunity and Immunotherapy,

a section of the journal

Frontiers in Immunology

Received: 14 March 2021

Accepted: 17 May 2021

Published: 04 June 2021

Citation:

Mao L, Qi Z, Zhang L, Guo J and Si L (2021) Immunotherapy in Acral and Mucosal Melanoma: Current

Status and Future Directions.

Front. Immunol. 12:680407. doi: 10.3389/fimmu.2021.680407

\section{Immunotherapy in Acral and Mucosal Melanoma: Current Status and Future Directions}

\author{
Lili Mao ${ }^{1 \dagger}$, Zhonghui Qi ${ }^{1 \dagger}$, Li Zhang ${ }^{2}$, Jun Guo ${ }^{1 *}$ and $\mathrm{Lu} \mathrm{Si}^{{ }^{1 *}}$ \\ ${ }^{1}$ Department of Melanoma, Peking University Cancer Hospital and Institute, Beijing, China, ${ }^{2}$ Global Medical Affairs, MSD \\ China, Shanghai, China
}

Acral and mucosal melanomas are extremely rare in Caucasians; however, they are the predominant melanoma subtypes in Asians and other non-Caucasian populations. Acral and mucosal melanomas share many clinicopathological features, including aggressive phenotypes, similar genetic landscapes, and grim prognoses. In spite of advances in melanoma management, patients with acral and mucosal melanomas show limited benefit from current therapies. The rarity of these subtypes of melanoma is a significant factor contributing to the poor understanding of these pathological subtypes and the lack of effective interventions. Furthermore, the mechanisms contributing to disparities between different types of melanoma remain largely unclear. Herein, we comprehensively review current knowledge on the clinicopathological characteristics and mutational landscapes of acral and mucosal melanomas, as well as providing an overview of current therapies for patients with these aggressive melanoma subtypes, focusing on available immunotherapeutic interventions. We also discuss pathological differences between different melanoma subtypes and summarize current knowledge on melanoma disparities between Asians and Caucasians. Finally, we discuss emerging immunotherapeutic strategies for the treatment of acral and mucosal melanomas, focusing on combination therapies with immune checkpoint inhibitors. Unraveling the unique features of acral and mucosal melanomas is key for their early diagnosis and for the development of effective therapies.

Keywords: acral melanoma, mucosal melanoma, immunotherapy, immune checkpoint inhibitors, combination therapy

\section{INTRODUCTION}

Melanoma is a type of skin cancer arising from melanocytes, the pigment-producing cells found in the epidermis, hair follicles, and iris, among other tissues. Although melanomas most frequently develop in sun-exposed areas of the skin (e.g., the chest, neck, back, and legs), they may also develop in the eyes and in parts of the body that are not exposed to the sun. Comprising only around 1\% of skin cancer cases, melanoma is far less common than other types of skin cancer, such as basal cell carcinoma and squamous cell carcinoma (SCC) (1). In most countries, the incidence of melanoma has been increasing over the past decades (2). Factors behind this rise in incidence rates include 
increased implementation of skin cancer screening programs, increased UV exposure, the rising popularity of indoor tanning, an increased number of skin biopsies being conducted, improved public awareness regarding suspicious pigmented lesions, and an increase in the number of specialist clinics for melanoma (3-7).

According to the American Cancer Society's estimates, approximately 106,110 new melanomas (31.91 per 100,000 person-years) will be diagnosed in the US in 2021 (8). In China, although the incidence of melanoma is lower than Western countries, it is growing at an annual rate of $3 \%-5 \%$ (9). The 2017 global burden of disease study revealed that the age-standardized rate of melanoma in China in 2017 was 0.9 per 100,000 person-years (10). Ancestral differences in the incidence rate of melanoma are particularly evident in the US, where populations are composed of individuals of diverse ancestral backgrounds. Calculations based on historical data from the US SEER database reported age-adjusted incidence rates of in situ melanoma per 100,000 person-years of 9.19 for non-Hispanic Whites, 1.26 for Hispanic Whites, 0.16 for African Americans, and 0.34 for Asians and Pacific Islanders (11). Furthermore, despite its rarity, melanoma is the leading cause of skin cancerrelated death (8). The poor prognosis of melanoma is primarily due to the high metastatic ability of melanoma cells (12).

There are different melanoma subtypes that originate from various parts of the body and exhibit differential clinicopathological and histological characteristics. On the basis of the anatomical location and the degree of sun-induced damage, melanomas can be classified into four primary subtypes: skin melanomas without chronic sun-induced damage, skin melanomas with chronic suninduced damage, mucosal melanomas, and acral melanomas (13, 14). Skin melanomas are collectively referred to as cutaneous melanomas (15). Acral melanomas account for around 1.0\%$1.5 \%$ of melanoma cases in the overall US population, in whom the vast majority (over 90\%) of melanomas are cutaneous $(16,17)$. In addition, mucosal melanoma is a rare melanoma subtype among Caucasians, accounting for only $\sim 1 \%-2 \%$ of all melanoma cases $(16,18,19)$. In sharp contrast, acral $(42 \%-65 \%)$ and mucosal (20\%-30\%) melanomas are the predominant melanoma subtypes in China and in other Asian countries (11, 20-26).

Accumulating evidence suggests that epidemiological, anatomical, and clinical differences may exist among melanoma patients with different pathological subtypes (27, 28 ); disparities in the prevalence of pathological subtypes and risk factors among melanoma patients have also been reported $(2,29)$. In addition, treatment response and survival outcomes differ considerably among patients with different melanoma subtypes $(30,31)$. While the mechanisms underlying these disparities remain to be elucidated, genetic and environmental contributors are believed to exist $(27,32,33)$.

Though causing significant mortality and exhibiting unique biological and clinical features, due to their relative rarity (compared with cutaneous melanomas) in Europe and North America, acral and mucosal melanoma are often overlooked and studies are scarce. Hence, our understanding of these melanoma subtypes remains limited, hindering the establishment of consensus on their optimal management. In this article, we review the current knowledge on acral and mucosal melanomas and discuss disparities in their clinicopathological features, mutational landscapes, tumor immune microenvironment, and treatment outcomes. We also summarize emerging treatment strategies, especially combination therapies with immune checkpoint inhibitors, for these rare melanoma subtypes. This comprehensive overview of acral and mucosal melanomas aims to facilitate the establishment of consensus guidelines on the optimal management of these rare melanoma subtypes, improve early diagnosis, and guide the development of more effective therapies based on their unique characteristics. All these are key to improving the survival of patients with acral or mucosal melanoma.

\section{ACRAL MELANOMA}

\section{Clinicopathological Characteristics}

Acral melanomas, also known as acral lentiginous melanomas, develop on non-hair-bearing skin, including the palms of the hands, the soles of the feet, or under the nails (34). The clinicopathological characteristics of acral melanomas differ significantly from those of cutaneous melanomas, with acral melanomas being more aggressive than cutaneous melanomas, regardless of the ancestry of patients (35). Importantly, patients with acral melanoma tend to be older and have fewer atypical nevi and a lower incidence of sunburn than patients with cutaneous melanoma (26). Furthermore, ulcerations and thick lesions are particularly common among Asian patients with acral melanoma (11, 36, 37). Importantly, melanoma thickness (Breslow depth) and ulceration are among the most significant factors associated with poor survival outcomes $(37,38)$. However, the higher tumor thickness and ulceration rates in Asian patients with melanoma compared with their Caucasian counterparts may reflect differences in the clinicopathological characteristics of acral and cutaneous melanomas, such as the higher aggressiveness of acral melanomas (35). In a recent study of 1157 Chinese patients with acral melanoma, Wei et al. (39) showed that patient prognosis varied depending on the anatomical location of the primary tumor. The study also showed that initial tumor stage and ulceration were significant predictors of melanoma-specific survival. Intriguingly, patients with primary melanomas on the soles of their feet had a worse prognosis than those with primary lesions on the palms and under the nails (39).

Additionally, ancestry-related disparities in clinicopathological characteristics have been reported among patients with acral melanoma. The 5-year disease-specific survival (DSS) of $53.5 \%$ in Chinese patients with acral melanoma reported by Lv et al. (25) was considerably lower than the 5 -year DSS of 70\% in Caucasians reported by Bello et al. (40) and of $67 \%$ in a predominantly white US cohort reported by Behbahani et al. (41). Similarly, Bradford et al. (30) reported that, among different ethnic groups with acral melanoma in the US, the 5-year and 10-year DSS rates were low in Hispanic Whites (72.8\% and 57.3\%) and Asian/Pacific Islanders (70.2\% and 54.1\%), intermediate in Blacks (77.2\% and 71.5\%), and 
high in non-Hispanic Whites (82.6\% and 69.4\%) (30). However, these differences in 5- and 10-year DSS rates among the different ancestral groups were not statistically significant after adjusting for tumor thickness or stage (30). Wang et al. (11) reported that among Asians/Pacific Islanders, Black, non-Hispanic White, and Hispanic White patients with acral melanoma, Asians and Pacific Islanders presented the thickest tumors. Moreover, Bradford et al. (30) noted that acral melanoma diagnosis at stage III was more common in Asians and Pacific Islanders than in non-Hispanic Whites, Hispanic Whites, and Blacks. Collectively, the findings of these studies suggest that factors including advanced disease stage, ulceration, and high lesion thickness at diagnosis may contribute to the poor survival outcomes observed for Asian patients with acral melanoma.

\section{Mutational Landscape}

As acral melanomas develop in sun-shielded areas of the body, they are not believed to be caused by exposure to ultraviolet (UV) radiation. Hence, oncogenic pathway activation in acral melanoma is UV-independent, in contrast to the induction of oncogenic circuits in cutaneous melanomas $(42,43)$. Wholeexome and whole-genome sequencing studies revealed that, compared with cutaneous melanomas, acral melanomas have fewer single nucleotide polymorphisms (SNPs) and indel mutations and a higher number of structural rearrangements and focal chromosomal copy number aberrations (44). Genes frequently mutated in cutaneous melanoma include $B R A F(45 \%-$ $50 \%$ of tumors), CDKN2A (13\%-40\%), NRAS ( 30\%), and TP53 (15\%-18\%), while BRAF (10\%-35\%), NRAS (8\%-22\%), and NF1 $(11 \%-23 \%)$ are often mutated in acral melanomas $(42,45)$. However, the frequency of BRAF and NRAS mutations in acral melanoma is considerably lower than that in cutaneous melanoma, leaving most acral melanoma patients ineligible for treatment with BRAF inhibitors and the combination of BRAF/ MEK inhibitors $(13,46)$.

A study of 514 acral melanomas revealed that alterations in the CDK4 pathway were frequent and that these alterations promoted G1 to S cell cycle transition and tumor progression. Notably, $82.7 \%$ of all samples harbored mutations in at least one of either $C D K 4, C C N D 1$, or $P 16^{\text {INK4a }}$ (47). mTOR mutations are also common in melanoma. Kong et al. analyzed 412 melanoma samples and found that $10.4 \%(n=43 / 412)$ of the samples had nonsynonymous mutations in mTOR. Notably, mTOR mutations were more frequent in acral melanomas (11.0\%) than in chronically sun-damaged melanomas (6.7\%). They also found that mTOR nonsynonymous mutations were significantly associated with poor survival in patients with melanoma, suggesting that PI3K-AKT-mTOR pathway inhibitors may represent a promising treatment strategy for patients with mTOR-mutant melanoma (48).

Gene mutations affecting the MAPK pathway, including those in $C-K I T$ and platelet-derived growth factor receptor alpha (PDGFRA), are more common in acral melanoma than in cutaneous melanoma (49). Interestingly, Dai et al. (50) found that the SNP rs2228230:T in PDGFRA resulted in lower PDGFRA expression levels and downstream signaling activity and was associated with favorable survival in patients with acral melanoma (50).

Additionally, compared with cutaneous melanomas [the predominant melanoma subtype in Caucasians] acral melanomas possess a higher number of chromosomal structural aberrations and copy number variations (CNV) and exhibit a low tumor mutational burden (TMB) $(42,45)$. Although ancestryrelated disparities in the TMB have been reported for different tumor types, including lung cancer (51-53), it remains unclear whether Asian patients with acral melanoma exhibit lower TMB than their Caucasian counterparts.

In a multi-fluorescence in situ hybridization study, the frequency of CCND1 amplification in 44 Chinese patients with acral melanoma was $45.4 \%$ (54). Mutations in telomerase reverse transcriptase (TERT), the gene encoding an enzyme crucial for telomere maintenance, also play a crucial role in melanoma development, with up to $50 \%$ of cutaneous melanomas harboring TERT promoter mutations $(55,56)$. However, a retrospective study of Chinese patients with acral melanoma showed that TERT promoter mutations were present in only $~ 5 \%$ of cases (57). In Caucasians, acral melanomas have been found to have a higher number of CNVs than cutaneous melanomas $(42,45)$. In contrast, in a Chinese cohort, a higher number of CNV amplifications were detected in non-CSD cutaneous melanomas than in acral melanomas (58). However, future molecular studies in patients with different ancestries are required to elucidate the relationship between ancestry and the mutational landscape of acral melanoma.

Data on the association between mutational profiles and response to treatment in patients with acral melanoma are relatively scarce. However, one recent study of 178 Asian patients with advanced melanoma (40\% with acral melanoma) who received treatment with immune checkpoint inhibitors suggested that NRAS mutations, TP53 mutations and NF2 deletions are associated with resistance to checkpoint inhibitors, whereas MYC and RPS6KB1 amplifications were observed more frequently in patients who responded to these treatments (59).

\section{Tumor Immune Microenvironment}

The PD-1/PD-L1 axis is one of the most well-studied mechanisms contributing to immune evasion in melanoma and other solid malignancies. Cancer cells and other cells in the tumor microenvironment (TME) can express PD-L1, and PD-L1 levels in the TME strongly predict response to immunotherapy and survival outcomes in patients with cutaneous melanoma (60). Specifically, high PD-L1 expression in the TME was associated with increased objective response rate (ORR) and improved progression-free survival (PFS) and overall survival (OS) rates in patients with melanoma treated with antiPD-1/PD-L1 monotherapy (61). Notably, PD-L1 expression in the TME is relatively low in acral melanomas. Kaunitz et al. (62) performed immunohistochemical staining for PD-L1 on formalin-fixed paraffin-embedded specimens from patients with melanoma and found that only $31 \%$ of acral melanomas expressed PD-L1 in the TME. 
Moreover, the poor prognosis of acral melanomas may, to some extent, be attributed to their poor immunogenicity and low mutational burden. Castaneda et al. $(63,64)$ demonstrated that low numbers of tumor-infiltrating lymphocytes (TILs) were associated with poor prognosis in patients with acral melanoma. CD8+ T cells are particularly important for the elimination of cancer cells, especially in patients undergoing treatment with immune checkpoint inhibitors (ICIs). Edwards et al. (65) showed that CD103+ tumor-resident CD8+ T cells expanded significantly in immunotherapy-naïve patients treated with anti-PD-1 agents (pembrolizumab). They also showed that the levels of tumor-resident CD8+ T cells were more accurate predictors of melanoma-specific survival than the levels of total CD8+ T cells. Interestingly, in a follow-up study, Edwards et al. (66) found that, although the presence of tumor-resident CD8+ $\mathrm{T}$ cells was positively associated with OS, TMB and structural variations in acral and mucosal melanomas were not correlated with the densities of tumor-infiltrating CD8+ T cells and other innate and adaptive immune cell types (66).

Comparison of whole-transcriptome and whole-genome sequencing data between Western and Asian melanoma patients indicated that gene signatures associated with antigen presentation and $\mathrm{T}$ cell inflammation were expressed in lower levels in Chinese patients than in Western patients $(31,67,68)$. Moreover, comprehensive molecular analyses of melanomas from 152 Asian patients (58 with recurrent or metastatic acral melanoma) revealed a significant association between a high neutrophil-lymphocyte ratio (NLR) and the poor survival outcomes of Asian patients undergoing treatment with PD-1 inhibitors (69). The role of neutrophils in the clinical efficacy of ICIs in patients with acral melanoma remains to be determined.

\section{Current Treatment Options}

Evidence on the clinical efficacy of different systemic treatments for acral melanoma from international, large-cohort studies is scarce due to the low incidence rate of acral melanoma. Thus, the establishment of standardized, effective systemic treatments remains an unmet clinical need. In a randomized phase II trial, 158 high-risk Chinese patients with acral melanoma were randomly assigned to receive adjuvant treatment with highdose IFN- $\alpha-2 b$ (HDI) for four weeks or one year. The median relapse-free survival (RFS) for 4-week HDI and 1-year HDI was 17.9 months and 22.5 months, respectively; this difference in RFS was not statistically significant (70). However, the effects of HDI on OS remain controversial (71).

The efficacy of targeted therapies has also been assessed in patients with acral melanoma. Aberrations in MAPK, PI3K/ AKT/PTEN, TERT, WNT, and CDK4/CDKN2A signaling pathways are frequent in acral melanoma (43). However, despite the fact that acral melanomas have potentially actionable targets (45), a limited number of targeted therapies are available for the treatment of patients with acral melanoma. The combination of BRAF and MEK inhibitors provides robust clinical responses in patients with $B R A F$-mutated melanoma (72-74). Nonetheless, the frequency of $B R A F$ mutations in acral melanoma is relatively low, limiting the clinical benefit of BRAF and MEK inhibitors in this patient population $(13,46)$. The feasibility of using PI3K/Akt/mTOR inhibitors (75), CDK inhibitors (76), or MDM2/p53 inhibitors (77) to treat acral melanoma is currently being investigated.

Since 2011, various ICIs have been approved by the US FDA and the Chinese National Medical Products Administration (NMPA) for use as monotherapy or in combination with other therapies in melanoma. These agents include ipilimumab, pembrolizumab, and nivolumab (78). The findings of multiple clinical trials indicate that, in Caucasian patients with advanced melanoma, response rates after PD-1 blockade with nivolumab or pembrolizumab range between $26 \%$ and $44 \%$. Compared with ipilimumab plus dacarbazine, monotherapy with nivolumab or pembrolizumab provided superior clinical outcomes in patients with advanced melanoma (79-81). However, results from patients with acral melanoma were seldom reported separately in these early clinical studies because of the rarity of this pathological subtype. Only one global, multi-center study (NCT02156804) involved sub-analysis of efficacy in patients with acral melanoma, who represented only 5.5\% $(n=55)$ of the total cohort (Table 1). Caucasian patients with acral melanoma had similar survival outcomes to those with nonacral cutaneous melanoma (82). Similarly, in 164 Asian patients with metastatic melanoma, there were no significant differences in clinical responses to ICIs based on melanoma subtypes (59). However, results from clinical trials and retrospective studies are conflicting.

Shoushtari et al. (83) reported an ORR of 32\% [95\% confidence interval (CI), 15\%-54\%] and a median PFS of 4.1 months in 25 patients with acral melanoma after treatment with

TABLE 1 | Summary of immunotherapy clinical trials involving sub-analysis of patients with acral melanoma.

\begin{tabular}{|c|c|c|c|c|c|c|}
\hline Trial identifier & Phase & Number of patients & Location & Treatment & Line of treatment & Results \\
\hline NCT02156804 & $\|$ & $\begin{array}{l}n=1,008 \text { (total) } \\
n=55(A M)\end{array}$ & Europe & Nivolumab & $2+$ & Median OS: 25.8 months (95\% Cl, 15.1-30.6 months) \\
\hline NCT02821000 & $\mathrm{lb}$ & $\begin{array}{l}\mathrm{n}=102(\text { total) } \\
\mathrm{n}=39(\mathrm{AM})\end{array}$ & China & Pembrolizumab & 2 & ORR, 15.8\% (95\% Cl, 6.0\%-31.3\%) \\
\hline NCT03013101 & $\|$ & $\begin{array}{l}\mathrm{n}=128 \text { (total) } \\
\mathrm{n}=50(\mathrm{AM})\end{array}$ & China & Toripalimab & 2 & ORR, $14.3 \%$ (95\% Cl, 5.9\%-27.2\%) \\
\hline JapicCTI-142533 & $\|$ & $\begin{array}{l}\mathrm{n}=24(\text { total }) \\
\mathrm{n}=7(\mathrm{AM})\end{array}$ & Japan & Nivolumab & 1 & ORR, 28.6\% (90\% Cl, 10.0\%-59.1\%) \\
\hline JapicCTI-152869 & $\|$ & $\begin{array}{l}\mathrm{n}=30(\text { total }) \\
\mathrm{n}=7(\mathrm{AM})\end{array}$ & Japan & Nivolumab \pm ipilimumab & 1 & ORR, 42.9\% (95\% Cl, 9.9\%-81.6\%) \\
\hline
\end{tabular}

AM, acral melanoma; ORR, objective response rate; OS, overall survival. 
pembrolizumab or nivolumab. A single-center study of 428 patients with metastatic melanoma (cutaneous in 283, unknown in 55, mucosal in 38, and acral in 22) showed that the median OS of patients with acral melanoma was significantly shorter than that of patients with cutaneous melanoma (17 months vs. 45 months; $P=0.047$ ) after immunotherapy with CTLA-4, PD-1, or PD-L1 inhibitors (84). Low PD-L1 expression in the TME and low numbers of TILs may contribute to the poor response of acral melanomas to ICIs. The low TMB of acral melanomas may also contribute to the poor outcomes in patients with acral melanomas undergoing treatment with ICIs. Tumors with a high TMB often exhibit superior clinical responses to ICIs versus tumors with a low $\operatorname{TMB}(85,86)$. A higher TMB leads to a higher load of neoantigens that may elicit antitumor immune responses during immunotherapy with ICIs $(87,88)$.

Importantly, increasing evidence suggests that Asian patients with acral melanomas have even lower rates of response to ICI than their Caucasian counterparts. A recent retrospective study of 193 Japanese patients with advanced acral melanoma (palm and sole, $\mathrm{n}=123$; nail apparatus, $\mathrm{n}=70$ ) showed that PD-1 blockade with nivolumab or pembrolizumab provided ORRs of $21.1 \%$ in the palm and sole group and $8.6 \%$ in the nail apparatus group; median OS was 22.3 months and 12.8 months, respectively (89). The study also showed that the ORR in the palm and sole group was higher in the $B R A F$ wild-type group than in the $B R A F$-mutant group (20.7\% vs. $8.1 \% ; P=0.04)(89)$. Similar findings were reported in the Chinese cohort of the openlabel, non-randomized, multicenter KEYNOTE-151 study evaluating the safety and efficacy of pembrolizumab (90). The KEYNOTE-151 study showed that in 103 Chinese patients with advanced or metastatic melanoma (39 with acral melanoma) previously treated with one line of therapy, pembrolizumab provided an overall ORR of $16.7 \%$ (95\% CI, 10.0\%-25.3\%), a median PFS of 2.8 months (95\% CI, 2.7-3.5 months), and a median OS of 12.1 months (95\% CI, 9.6 months-not reached) (90). In a multicenter phase II study evaluating the safety and efficacy of the humanized anti-PD-1 antibody toripalimab in 128 Chinese patients with advanced melanoma (POLARIS-01), the ORR in 50 toripalimab-treated patients with acral melanoma was $14.0 \%$, the median OS was 16.9 months, and the median PFS was 3.2 months (67). Additionally, NRAS mutations and CCDN1 amplifications, which are particularly common among Chinese patients with melanoma, were associated with poor response to toripalimab treatment (ORRs of $6.3 \%$ and $0 \%$, respectively). In a recent study, Byeon et al. (59) reported an overall response rate of $43.1 \%$ in 65 Asian patients with acral melanoma after first-line or second-line treatment with ICIs (pembrolizumab, nivolumab, or ipilimumab). These reported clinical outcomes in Asian patients in response to ICIs are considerably worse than those reported in Western populations $(83,91,92)$.

Given the role of the TMB and tumor immune micro environment in clinical responses to immunotherapy and targeted therapies, the molecular differences between melanomas in Asian and Caucasian patients may be critical drivers of any disparities in treatment response between these populations. In addition, genetic alterations in CDK4 pathway components
(CDK4, CCND1, and CDKN2A), which are particularly common in Asians, have been associated with innate resistance to PD-1 blockade in patients with acral melanoma (93). Consistently, a recent panel-based next-generation sequencing analysis by Hilke et al. (94) showed that CDKN2A deletions or loss of heterozygosity, which are commonly found in non-Caucasian patients with melanoma, were significantly enriched in patients with progressing melanomas after treatment with ICIs (nivolumab, pembrolizumab, or nivolumab plus ipilimumab).

\section{MUCOSAL MELANOMA}

\section{Clinicopathological Characteristics and Patient Survival}

Mucosal melanomas arise from melanocytes in mucosal tissues, such as those in the nasal cavity, the mucous membrane lining the sinuses and mouth, the anus, and the vagina. More rarely, mucosal melanomas may also develop in the gastrointestinal tract, urinary tract, and gall bladder (95). Due to their anatomical location, mucosal melanomas are often diagnosed at an advanced stage, contributing to their aggressive phenotypes and dismal prognosis $(95,96)$. Compared with cutaneous melanomas, mucosal melanomas present more aggressive characteristics, including advanced TNM stage at diagnosis, high growth rate, and high metastatic potential $(97,98)$.

In the US, the most common anatomical location of mucosal melanomas is the head and neck $(31 \%-55 \%)$, followed by the anorectum (17\%-24\%) and the vulvovaginal (18\%-40\%) regions (19). Mucosal melanomas less frequently arise on the mucosal lining of the pharynx, larynx, urinary tract, cervix, and esophagus $(16,19)$. In a retrospective analysis of 706 Chinese patients with mucosal melanomas, Lian et al. (99) found that the lower gastrointestinal tract was the most common site of primary lesions (26.5\%), followed by the nasal cavity and paranasal sinuses $(23 \%)$, gynecological sites $(22.5 \%)$, oral cavity $(15 \%)$, urological sites (5\%), and the upper gastrointestinal tract (5\%). However, differences in the subclassification system based on the tumors' anatomical location complicate the comparison of anatomical sites of mucosal melanomas across studies. Lian et al. (99) found that patients with primary mucosal melanomas at the nasal and oral cavities, the gastrointestinal tract, and gynecological and urological sites had similar 1-year (88\%, $83 \%$, and $86 \%), 2$-year $(66 \%, 57 \%$, and $61 \%)$, and 5 -year (27\%, $16 \%$, and $20 \%)$ OS rates (99). These findings are further supported by a multivariate analysis of the same 706 patients included in the analysis by Lian et al. that found no association between OS and anatomical site, although it did reveal an association between factors including depth of tumor invasion, number of lymph node metastases and sites of distant metastases and OS (100). Interestingly, a population-based study of a racially diverse cohort in California showed that the most common anatomical site of mucosal melanoma was the anorectum in Asian/Pacific Islanders, the genitourinary tract in non-Hispanic Whites, and the head and neck in Hispanics (101). Differences were also observed in the stage of presentation of 
mucosal melanomas among the different ancestral groups. Notably, 55\% of mucosal melanomas in Asians and Pacific Islanders were diagnosed at a metastatic stage; in contrast, the percentage of mucosal melanomas diagnosed at a metastatic stage (locally or distally) was $49 \%$ in Hispanics, $47 \%$ in nonHispanic Blacks, and 45\% in non-Hispanic Whites (101).

\section{Mutational Landscape}

Like acral melanomas, mucosal melanomas are driven by chromosomal structural aberrations. Their mutational burden is relatively low, perhaps because they are typically not caused by exposure to UV radiation $(42,102-104)$. A key difference in the melanoma mutational profiles between Asians and Caucasians is that in Caucasians, most melanomas, which are predominantly cutaneous melanomas, are driven by UV-induced point mutations, including driver mutations in BRAF and NRAS (45). The findings of whole-genome studies suggest that mutations in SF3B1 are common among mucosal melanomas harboring driver mutations $(42,45)$. In mucosal melanomas, apart from driver mutations affecting the MAPK pathway (e.g., NRAS, BRAF, NF1, and $K I T$ ), mutations in CTNNB1 affecting Wnt/ $\beta$-catenin pathway activation have also been reported $(103,105)$. Mutations in Wnt/ $\beta$-catenin pathway components may contribute to the poor immunotherapy response of patients with advanced-stage mucosal melanoma $(106,107)$. Notably, Sheng et al. (108) found that nearly $10 \%$ of mucosal melanomas in Chinese patients had mutations in either GNAQ or GNA11. Importantly, these mutations were associated with poor prognosis, likely contributing to the less favorable survival outcomes of Chinese patients with melanoma compared with Caucasian patients (108).

In a recent study of 213 Chinese patients with mucosal melanoma, $\mathrm{Xu}$ et al. (109) found that mutations in cell cyclerelated genes were particularly common, with $47.0 \%$ and $27.7 \%$ of samples exhibiting amplifications in CDK4 and CCND1, respectively. Similarly, $P 16^{I N K 4 a}$ was deleted in $57.7 \%$ of mucosal melanomas. Hence, alterations in CDK4 signaling components may predict response to CDK4 inhibitors in patients with mucosal melanoma (109). MicroRNAs may also have a predictive value in mucosal melanoma. Ma et al. (110) identified microRNA-23a-3p as a key tumor-suppressor in mucosal melanoma, inhibiting mucosal melanoma progression by targeting adenylate cyclase 1 (ADCY1) and thereby inhibiting CAMP and MAPK signaling (110).

Similar to acral melanoma, there are limited data describing the association between mutational profiles and response to treatment in mucosal melanoma. However, a study of 178 Asian patients with advanced melanoma (26\% with mucosal melanoma) receiving immune checkpoint inhibitors showed that NRAS mutations, TP53 mutations and NF2 deletions are associated with resistance to checkpoint inhibitors, with MYC and RPS6KB1 amplifications more frequent in patients responding to treatment with immune checkpoint inhibitors (59).

\section{Tumor Immune Microenvironment}

Little is known about the immune microenvironment of mucosal melanoma, although it is believed to be more tolerogenic than that of other melanoma subtypes (18). Similar to acral melanomas, mucosal melanomas express lower levels of PD-L1 in the TME than cutaneous melanomas. Kaunitz et al. (62) demonstrated that, although $62 \%$ of chronic sun-damaged melanomas expressed PD-L1 in the TME, only $44 \%$ of mucosal melanomas exhibited PD-L1 expression. Analysis of the TCGA dataset revealed that the transcript levels of PD-L1 in melanoma were lower in Asian patients than in Caucasian patients (111); nevertheless, it remains unclear whether mucosal or acral melanomas in Asian patients express lower PD-L1 levels than those in Caucasian patients. Interestingly, compared with Caucasians, Asian patients with melanoma tend to express lower levels of genes associated with antigen presentation and $\mathrm{T}$ cell inflammation $(31,67,68)$. Impaired antigen presentation and $\mathrm{T}$ cell infiltration may significantly contribute to the inferior response rates and survival outcomes of Asian patients with mucosal melanoma undergoing treatment with ICIs. Intriguingly, a retrospective analysis of 152 Asian patients with recurrent or metastatic melanoma (47 with mucosal melanoma) treated with anti-PD-1 agents indicated that a high NLR in the TME was associated with poor response and patient survival (69). Future studies are required to confirm the role of NLR as a biomarker predicting ICI resistance in mucosal melanoma.

\section{Current Systemic Treatment Options}

Mucosal melanomas are typically detected at an advanced stage due to their challenging anatomic location. Owing to the anatomic constraints of mucosal melanomas and the multifocal growth of the lesions, complete resection and wide negative margins are challenging to achieve, leading to a high recurrence rate following surgical management $(18,112)$. Chemotherapies have similar effects in cutaneous melanoma and mucosal melanoma but failed to significantly improve outcomes. In a multicenter retrospective study, Yi et al. (113) showed that the OS of patients with mucosal melanoma after first-line treatment with dacarbazine-based chemotherapy was significantly shorter than that of patients with cutaneous or acral melanoma (113). As mucosal melanoma is one of the most common melanoma subtypes in China, several Chinese clinical trials have been conducted to evaluate the safety and efficacy of different regimens in patients with mucosal melanoma. In a randomized phase II study, adjuvant therapy with six cycles of cisplatin $\left(75 \mathrm{mg} / \mathrm{m}^{2}\right)$ plus temozolomide $\left(200 \mathrm{mg} / \mathrm{m}^{2}\right)$ for resected mucosal melanoma with adventitial invasion or nodal metastases provided a significant survival benefit (114). Yan et al. (115) conducted a randomized phase II study in patients with advanced, previously untreated mucosal melanoma. Although first-line chemotherapy (carboplatin plus paclitaxel) combined with antiangiogenic therapy (bevacizumab) did not significantly improve the ORR compared with chemotherapy alone (19.7\% vs. 13.2\%, $P=0.384$ ), median PFS (4.8 vs. 3.0 months; HR, 0.461; 95\% CI, 0.306-0.695; $P<0.001)$ and median OS (13.6 vs. 9.0 months; HR, 0.611; 95\% CI, 0.407-0.917; $P=0.017$ ) were significantly improved in patients treated with combination therapy (115).

The efficacy and safety of targeted therapies, especially of agents targeting c-KIT, have also been investigated in patients with mucosal melanoma. The frequency of BRAF mutations in mucosal melanoma is low $(105,116)$; hence, most patients with mucosal melanoma do not benefit from BRAF inhibitors alone or in combination with MEK inhibitors $(117,118)$. On the other hand, activating mutations in KIT are relatively common in 
mucosal melanoma, being found in approximately $40 \%$ of all patients (119). However, in patients with KIT-mutated metastatic mucosal melanoma, agents targeting c-KIT failed to provide durable responses (120). In a phase II trial evaluating the efficacy of imatinib in patients with metastatic melanoma [17/24 (71\%) patients with mucosal melanoma] harboring activating KIT mutations or amplifications, imatinib provided an overall response rate of $29 \%$ and an overall disease control rate of $50 \%$. However, disease control rates varied depending on the KIT status, with patients with KIT-mutant melanoma gaining a higher benefit than those with KIT amplification (121). Another phase II trial involving patients with melanoma [12/ 19 (74\%) patients with mucosal melanoma] harboring KIT mutations or amplifications showed that nilotinib may benefit patients with KIT alterations and whose tumors progressed after treatment with imatinib; however, patients with brain metastasis did not benefit from the treatment (122).

Advances in immunotherapies have provided promising therapeutic approaches for patients with advanced mucosal melanoma. Nevertheless, clinical studies suggest that patients with mucosal melanomas tend to be less responsive to ICIs than those with cutaneous melanomas (Table 2). This holds true for both Asian and Caucasian patients $(123,124)$. In a study of 35 patients with mucosal melanoma, monotherapy with pembrolizumab or nivolumab provided an ORR of 23\% (95\% CI, 10\%-40\%) and a median PFS of 3.9 months (83). In a singlecenter study of 428 patients with metastatic melanoma, Klemen et al. (84) demonstrated that immunotherapy with CTLA-4, PD-1, or PD-L1 inhibitors provided a significantly shorter median OS in patients with mucosal melanoma than in those with cutaneous melanoma (18 vs. 45 months; $P=0.003$ ). However, in a recent study of 164 Asian patients treated with pembrolizumab, nivolumab, or ipilimumab for metastatic melanoma, response rates in patients with mucosal melanoma (41.9\%) were not significantly different from those in patients with acral $(43.1 \%)$ or cutaneous melanoma (54.7\%) (59).

A post-hoc analysis of three trials (KEYNOTE-001, KEYNOTE-002, and KEYNOTE-006) involving 1567 patients with melanoma (84 with advanced mucosal melanoma) revealed that the ORR in patients treated with pembrolizumab was $19 \%$ (95\% CI, 11\%-29\%), the median PFS was 2.8 months (95\% CI, 2.7-2.8), and the median OS was 11.3 months (95\% CI, 7.7-16.6 months) (125). The 5-year follow-up of 79 patients with mucosal melanoma from the CheckMate-067 study revealed that compared with ipilimumab alone, the combination of nivolumab and ipilimumab resulted in a considerably higher ORR ( $43 \%$ vs. $7 \%)$, complete response rate (14\% vs. $0 \%)$, and OS rate $(36 \%$ vs. $7 \%)$. However, clinical outcomes in patients with mucosal melanoma tended to be worse than those in the intentto-treat population (126). In addition, these different studies included patients with different baseline characteristics and cannot be easily directly compared head-to-head.

Notably, clinical outcomes in response to ICIs tend to be less favorable in Asian patients with mucosal melanomas than their Caucasian counterparts. The KEYNOTE-151 study demonstrated that in 15 Chinese patients with advanced or metastatic mucosal melanoma, pembrolizumab provided an overall ORR of $13.3 \%$ (95\% CI, $1.7 \%-40.5 \%)$ (90). The POLARIS-01 study showed that clinical outcomes in 22 Chinese patients with mucosal melanoma treated with toripalimab were very poor; the ORR was $0 \%$, the median OS was 10.3 months, and the median PFS was 1.9 months (67). Similarly, a retrospective analysis of 162 Chinese patients with advanced melanoma (121 with cutaneous and 41 mucosal) showed that the overall median PFS of patients with mucosal melanoma was 13 months; the median PFS in the immunotherapy group was 14 months and in the chemotherapy group was six months (127). In a German phase II study, ipilimumab provided an ORR of $17 \%$ in seven patients with metastatic mucosal melanoma, and the median OS was 9.6 months (95\% CI, 1.6-11.1 months) (128). A retrospective analysis of 75 German patients with mucosal melanoma showed that ipilimumab provided an ORR of 12.5 (median OS and PFS were not reached) (129). In addition, a pooled analysis of patients with mucosal melanoma from the US, Europe, and Australia revealed an ORR of 23.3\% (95\% CI, 14.8-33.6) and a

TABLE 2 | Summary of immunotherapy clinical trials involving sub-analysis of patients with mucosal melanoma.

\begin{tabular}{|c|c|c|c|c|c|c|}
\hline Trial identifier & Phase & $\begin{array}{l}\text { Number of } \\
\text { patients }\end{array}$ & Location & Treatment & $\begin{array}{l}\text { Line of } \\
\text { treatment }\end{array}$ & Results \\
\hline NCT02156804 & $\|$ & $\begin{array}{l}\mathrm{n}=1,008(\text { total }) \\
\mathrm{n}=63(\mathrm{MM})\end{array}$ & Europe & Nivolumab & $2+$ & Median OS, 11.5 months (95\% Cl, 6.4-15.0 months) \\
\hline NCT02821000 & $\mathrm{lb}$ & $\begin{array}{l}\mathrm{n}=102(\text { total }) \\
\mathrm{n}=15(\mathrm{MM})\end{array}$ & China & Pembrolizumab & 2 & ORR, 13.3\% (95\% Cl, 1.7\%-40.5\%) \\
\hline NCT03013101 & $\|$ & $\begin{array}{l}\mathrm{n}=128(\text { total }) \\
\mathrm{n}=22(\mathrm{MM})\end{array}$ & China & Toripalimab & 2 & ORR, 0\% (95\% Cl, 0.0\%-17.6\%) \\
\hline NCT01844505 & III & $\begin{array}{l}\mathrm{n}=1,295(\text { total }) \\
\mathrm{n}=79(\mathrm{MM})\end{array}$ & International & $\begin{array}{l}\text { Nivolumab } \pm \\
\text { ipilimumab }\end{array}$ & 1 & $\begin{array}{l}\text { ORR, } 43 \% \text { with NIVO+IPI vs. } 30 \% \text { with NIVO and } 7 \% \text { with } \\
\text { IPI }\end{array}$ \\
\hline JapicCTI-142533 & $\|$ & $\begin{array}{l}\mathrm{n}=24(\text { total }) \\
\mathrm{n}=6(\mathrm{MM})\end{array}$ & Japan & Nivolumab & 1 & ORR, 33.3\% (90\% Cl, 11.7\%-65.3\%) \\
\hline JapicCTI-152869 & $\|$ & $\begin{array}{l}\mathrm{n}=30 \text { (total) } \\
\mathrm{n}=12(\mathrm{MM})\end{array}$ & Japan & $\begin{array}{l}\text { Nivolumab } \pm \\
\text { ipilimumab }\end{array}$ & 1 & ORR, 33.3\% (95\% Cl, 9.9\%-65.1\%) \\
\hline $\begin{array}{l}\text { DeCOG-MM-PAL11- } \\
\text { Trial }\end{array}$ & $\|$ & $\begin{array}{l}\mathrm{n}=103 \text { (total) } \\
\mathrm{n}=7(\mathrm{MM})\end{array}$ & Germany & Ipilimumab & $2+$ & Median OS, 9.6 months (95\% Cl, 1.6-11.1 months) \\
\hline
\end{tabular}

IPI, ipilimumab; MM, mucosal melanoma; NIVO, nivolumab; ORR, objective response rate; OS, overall survival. 
median PFS of 3.0 months (95\% CI, 2.2-not reached) after treatment with nivolumab (124). Similarly, in 84 patients with advanced mucosal melanoma from North America, Europe, and Australia, pembrolizumab provided an ORR of 19\% (95\% CI, 11-29), a median PFS of 2.8 months (95\% CI, 2.7-2.8), and a median OS of 11.3 months (95\% CI, 7.7-16.6) (125). A multicenter retrospective study conducted in France revealed that in 151 patients with mucosal melanoma, treatment with anti-CTLA-4 or anti-PD-1 antibodies provided an ORR of $11.9 \%$ (95\% CI, 7.2-18.2) and a median OS of 15.97 months (interquartile range, 6.89-27.11 months) (130). Shoushtari et al. conducted a retrospective cohort analysis of 35 patients with mucosal melanoma treated with pembrolizumab or nivolumab in the US. They found that PD-1 blockade provided an ORR of $23 \%$ (95\% CI, 10-40), a median PFS of 3.9 months, and a median OS of 12.4 months (83).

Differences in the prevalence of mutations affecting $\mathrm{Wnt} / \beta$ catenin pathway activation among different melanoma subtypes (105) may contribute to the unfavorable outcomes of Chinese patients with mucosal melanoma treated with ICIs. Additionally, the high frequency of KIT mutations in Asians $(131,132)$ may contribute to the low response rates of Asians with mucosal melanoma undergoing treatment with targeted therapies.

\section{FUTURE DIRECTIONS FOR THE TREATMENT OF ACRAL AND MUCOSAL MELANOMAS: COMBINATION THERAPIES WITH ICls}

Despite the significant success of ICI monotherapy in the management of advanced cutaneous melanoma, ICI monotherapy is less effective in patients with acral and mucosal melanoma. The difference in response to ICIs may partially be explained by differences in the TMB and immune microenvironment of the tumor. Thus, novel therapies, especially combination therapies, are needed to improve the long-term outcomes of patients with these two melanoma subtypes. Combination immunotherapies that target various phases of the cancer-immunity cycle may represent a promising strategy to overcome immune escape and prevent immunotherapy resistance. By targeting multiple mechanisms by which tumor cells evade immune surveillance, combination therapies may exert synergistic antitumor effects and improve long-term survival outcomes. Numerous ongoing trials are investigating the efficacy and safety of different combination therapies specifically in patients with acral or mucosal melanoma (Table 3).

A study of 20 patients with primary mucosal oral melanoma demonstrated that the expression level of vascular endothelial growth factor (VEGF) was associated with poor survival outcomes (133), suggesting VEGF to be a promising therapeutic target for the treatment of oral mucosal melanoma. Nevertheless, no significant improvement in survival outcomes was observed in patients with advanced melanoma treated with anti-angiogenic therapy alone compared with those treated with chemotherapy $(134,135)$. In addition to its role in neovascularization, VEGF signaling has also emerged as a critical immunosuppressive mechanism in the tumor microenvironment. In vivo findings from murine cancer models indicated that the combined inhibition of the VEGF receptor (VEGFR) and PD-1 synergistically increased the number of TILs and inhibits tumor growth (136). Preliminary findings of an ongoing clinical study evaluating the safety and efficacy of axitinib combined with toripalimab, a humanized antibody targeting PD-1, revealed that the combination therapy was tolerable and exhibited encouraging

TABLE 3 | Summary of ongoing immunotherapy trials for the treatment of acral and mucosal melanoma.

\begin{tabular}{|c|c|c|c|c|c|c|}
\hline $\begin{array}{l}\text { Trial } \\
\text { identifier }\end{array}$ & Phase & Target population & $\mathbf{n}$ & Regimens & Setting & Location \\
\hline $\begin{array}{l}\text { NCT03820986 } \\
(\text { LEAP-003) }\end{array}$ & III & Cutaneous, acral, and mucosal melanoma & 660 & $\begin{array}{l}\text { Pembrolizumab + lenvatinib vs. } \\
\text { pembrolizumab }\end{array}$ & First-line & International \\
\hline NCT03955354 & $\|$ & Acral melanoma & 30 & SHR-1210+ apatinib & First-line & China \\
\hline NCT03991975 & I, II & Metastatic and acral melanoma & 42 & TQB2450 + anlotinib & $\begin{array}{l}\text { Second- or later- } \\
\text { line }\end{array}$ & China \\
\hline NCT04331093 & $\|$ & Acral melanoma & 40 & SHR-1210+ apatinib & Neoadjuvant & China \\
\hline NCT04277663 & III & Acral melanoma & 300 & IBI310+ IBI308 vs IBI308 vs HDI & Adjuvant & China \\
\hline NCT04397770 & $\|$ & Acral melanoma & 40 & SHR-1210+apatinib+ temozolomide & First-line & China \\
\hline NCT03602547 & $\|$ & Mucosal melanoma & 40 & Toripalimab+CM082 & First-line & China \\
\hline NCT03241186 & $\|$ & Mucosal melanoma & 36 & Ipilimumab+nivolumab & Adjuvant & US \\
\hline NCT03986515 & $\|$ & Mucosal melanoma & 40 & Apatinib+SHR-1210 & $\begin{array}{l}\text { Second- or later- } \\
\text { line }\end{array}$ & China \\
\hline NCT04622566 & $\|$ & Mucosal melanoma & 26 & Pembrolizumab+lenvatinib & Adjuvant & China \\
\hline NCT04462965 & $\|$ & Mucosal melanoma & 294 & Toripalimab+temozolomide & Adjuvant & China \\
\hline NCT04318717 & I, II & Mucosal melanoma of head and neck & 16 & $\begin{array}{l}\text { Pembrolizumab+ hypofractionated } \\
\text { radiotherapy }\end{array}$ & Adjuvant & US \\
\hline NCT04180995 & $\|$ & Mucosal melanoma & 30 & Toripalimab, axitinib & Neoadjuvant & China \\
\hline NCT04091217 & $\|$ & Locally advanced or metastatic mucosal melanoma & 43 & Atezolizumab+bevacizumab & First- or later line & China \\
\hline NCT03941795 & $\|$ & Metastatic mucosal melanoma & 99 & Toripalimab+axitinib & First-line & China \\
\hline NCT02978443 & $\|$ & Metastatic acral and mucosal melanoma & 14 & Nivolumab+ipilimumab & First- or later line & US \\
\hline NCT04653038 & 1 & $\begin{array}{l}\text { Unresectable, recurrent, or metastatic acral and mucosal } \\
\text { melanoma }\end{array}$ & 160 & MGD013 (tebotelimab) & $\begin{array}{l}\text { Second- or later- } \\
\text { line }\end{array}$ & China \\
\hline
\end{tabular}


clinical efficacy in 29 treatment-naïve Asian patients with metastatic mucosal melanoma. The ORR was 48.3\% (95\% CI, $29.4 \%-67.5 \%)(137,138)$. The authors of this study (138) found no correlation between common driver mutations and clinical response to the combination therapy. However, PD-L1 expression and high TMB were significantly associated with high ORR and PFS, especially in PD-L1 positive patients (138).

Similarly, the phase Ib/II KEYNOTE-146 trial showed that pembrolizumab combined with lenvatinib exerted promising antitumor activity (ORR, 47.6\%; 95\% CI, 25.7-70.2) in patients with advanced melanoma (139). An international randomized phase III trial (LEAP-003, NCT03820986) of pembrolizumab combined with lenvatinib for the treatment of metastatic melanoma is currently ongoing and includes patients with acral and mucosal melanomas. Furthermore, in a phase II study of patients with advanced mucosal melanoma (NCT03602547) in our center, we found that the combination of toripalimab with the multi-target tyrosine kinase inhibitor vorolanib (CM082; $150 \mathrm{mg}$ ) provided an ORR of $22.2 \%$, a disease control rate of 55.5\%, and a median PFS of 5.7 months (140). Similar small clinical trials of combination regimens (e.g., ICI combined with anlotinib, apatinib, or bevacizumab) for the treatment of acral and mucosal melanoma are also ongoing.

The efficacy of ipilimumab plus nivolumab in patients with mucosal melanoma is also under investigation. A recent pooled analysis of CheckMate-067 and CheckMate-069 trials showed that, compared with patients treated with nivolumab monotherapy, 35 mucosal melanoma patients treated with the combination of nivolumab plus ipilimumab exhibited higher ORR (37.1\% vs. 23.3\%) and median PFS (5.9 vs. 3 months). However, severe toxicities were common among patients receiving the combination therapy (124). Response to ipilimumab plus nivolumab in patients with mucosal melanoma was associated with PD-L1 expression in tumor cells (141). In an open-label, single-arm, multicenter phase II study, first-line nivolumab combined with ipilimumab in Japanese patients with different types of unresectable or recurrent melanoma provided an ORR of $33.3 \%$ and a 1 -year OS rate of $83.3 \%$ (142). Other combination regimens, including ICI combined with temozolomide (an orally active congener of dacarbazine) and radiotherapy (mucosal melanoma of head and neck), have also been initiated or planned.

\section{CONCLUSIONS}

Acral and mucosal melanomas share numerous clinicopathological features, including a late onset, aggressive phenotypes, a broad radial growth phase with prominent lentiginous growth, the lack of driver mutations that are common in other melanoma subtypes, and poor prognosis. Despite recent advances in the treatment of melanoma, patients with these rare and aggressive melanoma subtypes show limited benefit from current therapies. Standardized and effective interventions for the treatment of patients with acral and mucosal melanomas are currently lacking. As acral and mucosal melanomas are highly aggressive, combination therapies are more likely to provide long-term clinical benefit. The combination of anti-angiogenic therapies with ICIs is among the most promising strategies to suppress the progression of acral and mucosal melanomas and improve long-term survival outcomes. The clinical efficacy and safety of such combination approaches are currently under extensive clinical investigation. Given the rarity of these melanoma subtypes, large multicentric prospective clinical trials are warranted to improve long-term outcomes in patients with acral and mucosal melanomas, the predominant pathological subtypes of melanoma in Asians and people of African ancestry.

Mounting evidence shows that, compared to patients with cutaneous melanomas, those with acral and mucosal melanomas respond less well to current and emerging immunotherapies and other systemic treatments. Furthermore, ancestral disparities in melanoma treatment outcomes and patient survival also seem to exist. However, evidence for these ancestry-related differences in melanoma treatment outcomes is mostly derived from retrospective population-based studies or comparisons of results from various small-cohort clinical trials involving patients that did not necessarily have the same baseline characteristics. Hence, welldesigned clinical trials involving baseline characteristic-matched patients with melanoma are required to confirm the ancestral disparities in acral and mucosal melanoma treatment outcomes. Enhancing the enrollment of racial minorities in clinical trials may also help develop effective therapies to treat aggressive melanoma subtypes that are rare among Caucasian patients.

Additionally, differences in the clinicopathological features, mutational profiles, and tumor immune microenvironments may contribute to disparities in melanoma treatment outcomes; hence, these factors should be taken into account in clinical decision making. Understanding the genetic and environmental determinants of melanoma disparities is paramount to facilitating early diagnosis, developing effective treatments, and improving survival outcomes in patients with acral and mucosal melanoma.

\section{AUTHOR CONTRIBUTIONS}

LM, ZQ, LZ, JG and LS contributed to the conception of the review, conduct of the literature review, manuscript drafting and approval of the final draft. LZ collected or assembled the data and provided substantive suggestions for revision or critically reviewed subsequent iterations of the manuscript. All authors contributed to the article and approved the submitted version.

\section{FUNDING}

This review was funded by MSD China.

\section{ACKNOWLEDGMENTS}

Editorial support for this manuscript was provided by Christos Evangelou, PhD (Rude Health Consulting) and was funded by MSD China. 


\section{REFERENCES}

1. Bradford PT. Skin Cancer in Skin of Color. Dermatol Nurs (2009) 21 (4):170-8.

2. Erdei E, Torres SM. A New Understanding in the Epidemiology of Melanoma. Expert Rev Anticancer Ther (2010) 10(11):1811-23. doi: 10.1586/era.10.170

3. Raimondi S, Suppa M, Gandini S. Melanoma Epidemiology and Sun Exposure. Acta Derm Venereol (2020) 100(11):adv00136. doi: 10.2340/ 00015555-3491

4. Brunssen A, Waldmann A, Eisemann N, Katalinic A. Impact of Skin Cancer Screening and Secondary Prevention Campaigns on Skin Cancer Incidence and Mortality: A Systematic Review. J Am Acad Dermatol (2017) 76(1):12939.e10. doi: 10.1016/j.jaad.2016.07.045

5. Gardner LJ, Strunck JL, Wu YP, Grossman D. Current Controversies in Early-Stage Melanoma: Questions on Incidence, Screening, and Histologic Regression. J Am Acad Dermatol (2019) 80(1):1-12. doi: 10.1016/ j.jaad.2018.03.053

6. Weyers W. The 'Epidemic' of Melanoma Between Under- and Overdiagnosis. J Cutan Pathol (2012) 39(1):9-16. doi: 10.1111/j.16000560.2011.01831.x

7. Weyers W. Screening for Malignant Melanoma-a Critical Assessment in Historical Perspective. Dermatol Pract Concept (2018) 8(2):89-103. doi: 10.5826/dpc.0802a06

8. Siegel RL, Miller KD, Fuchs HE, Jemal A. Cancer Statistics, 2021. CA Cancer J Clin (2021) 71(1):7-33. doi: 10.3322/caac.21654

9. Guo J, Qin S, Liang J, Lin T, Si L, Chen X, et al. Chinese Guidelines on the Diagnosis and Treatment of Melanoma (2015 Edition). Ann Transl Med (2015) 3(21):322. doi: 10.3978/j.issn.2305-5839.2015.12.23

10. Wu Y, Wang Y, Wang L, Yin P, Lin Y, Zhou M. Burden of Melanoma in China, 1990-2017: Findings From the 2017 Global Burden of Disease Study. Int J Cancer (2020) 147(3):692-701. doi: 10.1002/ijc.32764

11. Wang Y, Zhao Y, Ma S. Racial Differences in Six Major Subtypes of Melanoma: Descriptive Epidemiology. BMC Cancer (2016) 16:691. doi: 10.1186/s12885-016-2747-6

12. Damsky WE, Rosenbaum LE, Bosenberg M. Decoding Melanoma Metastasis. Cancers (Basel) (2010) 3(1):126-63. doi: 10.3390/cancers3010126

13. Curtin JA, Fridlyand J, Kageshita T, Patel HN, Busam KJ, Kutzner H, et al. Distinct Sets of Genetic Alterations in Melanoma. N Engl J Med (2005) 353 (20):2135-47. doi: 10.1056/NEJMoa050092

14. Huang K, Xu Y, Gabriel EM, Misra S, Chen Y, Bagaria SP. Comparative Analysis of Acral Melanoma in Chinese and Caucasian Patients. J Skin Cancer (2020) 2020:5169051. doi: 10.21203/rs.2.22789/v1

15. Thompson JF, Scolyer RA, Kefford RF. Cutaneous Melanoma. Lancet (2005) 365(9460):687-701. doi: 10.1016/S0140-6736(05)17951-3

16. Chang AE, Karnell LH, Menck HR. The National Cancer Data Base Report on Cutaneous and Noncutaneous Melanoma: A Summary of 84,836 Cases From the Past Decade. Am Coll Surgeons Commission Cancer Am Cancer Society Cancer (1998) 83(8):1664-78. doi: 10.1002/(SICI)1097-0142 (19981015)83:8<1664::AID-CNCR23>3.0.CO;2-G

17. Shaikh WR, Xiong M, Weinstock MA. The Contribution of Nodular Subtype to Melanoma Mortality in the United States, 1978 to 2007. Arch Dermatol (2012) 148(1):30-6. doi: 10.1001/archdermatol.2011.264

18. Goldemberg DC, Thuler LCS, de Melo AC. An Update on Mucosal Melanoma: Future Directions. Acta Dermatovenerol Croat (2019) 27 (1):11-5.

19. McLaughlin CC, Wu XC, Jemal A, Martin HJ, Roche LM, Chen VW. Incidence of Noncutaneous Melanomas in the U.S. Cancer (2005) 103 (5):1000-7. doi: 10.1002/cncr.20866

20. Bai X, Mao L, Guo J. Comments on. Chin J Cancer Res (2019) 31(5):740-1. doi: 10.21147/j.issn.1000-9604.2019.05.03

21. Teh YL, Goh WL, Tan SH, Yong G, Sairi ANH, Soo KC, et al. Treatment and Outcomes of Melanoma in Asia: Results From the National Cancer Centre Singapore. Asia Pac J Clin Oncol (2018) 14(2):e95-e102. doi: 10.1111/ ajco. 12802

22. Lee HY, Chay WY, Tang MB, Chio MT, Tan SH. Melanoma: Differences Between Asian and Caucasian Patients. Ann Acad Med Singap (2012) 41 (1):17-20.
23. Bellew S, Del Rosso JQ, Kim GK. Skin Cancer in Asians: Part 2: Melanoma. J Clin Aesthet Dermatol (2009) 2(10):34-6.

24. Krige JE. Melanoma in Black South Africans. S Afr J Surg (2010) 48(3):74-5, 99.

25. Lv J, Dai B, Kong Y, Shen X, Kong J. Acral Melanoma in Chinese: A Clinicopathological and Prognostic Study of 142 Cases. Sci Rep (2016) 6:31432. doi: 10.1038/srep31432

26. Piliang MP. Acral Lentiginous Melanoma. Clin Lab Med (2011) 31(2):281-8. doi: 10.1016/j.cll.2011.03.005

27. Cormier JN, Xing Y, Ding M, Lee JE, Mansfield PF, Gershenwald JE, et al. Ethnic Differences Among Patients With Cutaneous Melanoma. Arch Intern Med (2006) 166(17):1907-14. doi: 10.1001/archinte.166.17.1907

28. Byrd-Miles K, Toombs EL, Peck GL. Skin Cancer in Individuals of African, Asian, Latin-American, and American-Indian Descent: Differences in Incidence, Clinical Presentation, and Survival Compared to Caucasians. J Drugs Dermatol (2007) 6(1):10-6.

29. Qian Y, Johannet P, Sawyers A, Yu J, Osman I, Zhong J. The Ongoing Racial Disparities in Melanoma: An Analysis of the Surveillance, Epidemiology, and End Results Database (SEER) Database (1975-2016). J Am Acad Dermatol (2020) 84:1585-93. doi: 10.1016/j.jaad.2020.08.097

30. Bradford PT, Goldstein AM, McMaster ML, Tucker MA. Acral Lentiginous Melanoma: Incidence and Survival Patterns in the United States, 1986-2005. Arch Dermatol (2009) 145(4):427-34. doi: 10.1001/archdermatol.2008.609

31. Shoushtari AN, Bao R, Luke JJ. Pd-1 Blockade in Chinese Versus Western Patients With Melanoma. Clin Cancer Res (2020) 26(16):4171-3. doi: 10.1158/1078-0432.CCR-20-1558

32. Hu S, Parmet Y, Allen G, Parker DF, Ma F, Rouhani P, et al. Disparity in Melanoma: A Trend Analysis of Melanoma Incidence and Stage at Diagnosis Among Whites, Hispanics, and Blacks in Florida. Arch Dermatol (2009) 145 (12):1369-74. doi: 10.1001/archdermatol.2009.302

33. Harvey VM, Patel H, Sandhu S, Wallington SF, Hinds G. Social Determinants of Racial and Ethnic Disparities in Cutaneous Melanoma Outcomes. Cancer Control (2014) 21(4):343-9. doi: 10.1177/ 107327481402100411

34. Saida T. Acral Melanoma. DE Fisher and BC Bastian, editors. Melanoma. New York, NY: Springer New York (2018) p. 1-28.

35. Basurto-Lozada P, Molina-Aguilar C, Castaneda-Garcia C, Vázquez-Cruz ME, Garcia-Salinas OI, Álvarez-Cano A, et al. Acral Lentiginous Melanoma: Basic Facts, Biological Characteristics and Research Perspectives of an Understudied Disease. Pigment Cell Melanoma Res (2021) 34(1):59-71. doi: $10.1111 /$ pcmr.12885

36. Chang JW, Guo J, Hung CY, Lu S, Shin SJ, Quek R, et al. Sunrise in Melanoma Management: Time to Focus on Melanoma Burden in Asia. Asia Pac J Clin Oncol (2017) 13(6):423-7. doi: 10.1111/ajco.12670

37. Chi Z, Li S, Sheng X, Si L, Cui C, Han M, et al. Clinical Presentation, Histology, and Prognoses of Malignant Melanoma in Ethnic Chinese: A Study of 522 Consecutive Cases. BMC Cancer (2011) 11:85. doi: 10.1186/ 1471-2407-11-85

38. Pereira Cherobin ACF, Wainstein AJA, Colosimo EA, Goulart EMA Bittencourt FV. Prognostic Factors for Metastasis in Cutaneous Melanoma. Bras Dermatol (2018) 93(1):19-26. doi: 10.1590/abd18064841.20184779

39. Wei X, Wu D, Li H, Zhang R, Chen Y, Yao H, et al. The Clinicopathological and Survival Profiles Comparison Across Primary Sites in Acral Melanoma. Ann Surg Oncol (2020) 27(9):3478-85. doi: 10.1245/s10434-020-08418-5

40. Bello DM, Chou JF, Panageas KS, Brady MS, Coit DG, Carvajal RD, et al. Prognosis of Acral Melanoma: A Series of 281 Patients. Ann Surg Oncol (2013) 20(11):3618-25. doi: 10.1245/s10434-013-3089-0

41. Behbahani S, Malerba S, Samie FH. Acral Lentiginous Melanoma: Clinicopathological Characteristics and Survival Outcomes in the US National Cancer Database 2004-2016. Br J Dermatol (2020) 183(5):952-4. doi: 10.1111/bjd.19211

42. Rabbie R, Ferguson P, Molina-Aguilar C, Adams DJ, Robles-Espinoza CD. Melanoma Subtypes: Genomic Profiles, Prognostic Molecular Markers and Therapeutic Possibilities. J Pathol (2019) 247(5):539-51. doi: 10.1002/ path.5213

43. Tod BM, Schneider JW, Bowcock AM, Visser WI, Kotze MJ. The Tumor Genetics of Acral Melanoma: What Should a Dermatologist Know? JAAD Int (2020) 1(2):135-47. doi: 10.1016/j.jdin.2020.07.004 
44. Newell F, Wilmott JS, Johansson PA, Nones K, Addala V, Mukhopadhyay P, et al. Whole-Genome Sequencing of Acral Melanoma Reveals Genomic Complexity and Diversity. Nat Commun (2020) 11(1):5259. doi: 10.1038/ s41467-020-18988-3

45. Hayward NK, Wilmott JS, Waddell N, Johansson PA, Field MA, Nones K, et al. Whole-Genome Landscapes of Major Melanoma Subtypes. Nature (2017) 545(7653):175-80. doi: 10.1038/nature22071

46. Si L, Kong Y, Xu X, Flaherty KT, Sheng X, Cui C, et al. Prevalence of BRAF V600E Mutation in Chinese Melanoma Patients: Large Scale Analysis of BRAF and NRAS Mutations in a 432-Case Cohort. Eur J Cancer (2012) 48 (1):94-100. doi: 10.1016/j.ejca.2011.06.056

47. Kong Y, Sheng X, Wu X, Yan J, Ma M, Yu J, et al. Frequent Genetic Aberrations in the CDK4 Pathway in Acral Melanoma Indicate the Potential for CDK4/6 Inhibitors in Targeted Therapy. Clin Cancer Res (2017) 23 (22):6946-57. doi: 10.1158/1078-0432.CCR-17-0070

48. Kong Y, Si L, Li Y, Wu X, Xu X, Dai J, et al. Analysis of Mtor Gene Aberrations in Melanoma Patients and Evaluation of Their Sensitivity to PI3K-AKT-mTOR Pathway Inhibitors. Clin Cancer Res (2016) 22(4):101827. doi: 10.1158/1078-0432.CCR-15-1110

49. Kong Y, Si L, Zhu Y, Xu X, Corless CL, Flaherty KT, et al. Large-Scale Analysis of KIT Aberrations in Chinese Patients With Melanoma. Clin Cancer Res (2011) 17(7):1684-91. doi: 10.1158/1078-0432.CCR-10-2346

50. Dai J, Yang L, Xu T, Si L, Cui C, Sheng X, et al. A Functional Synonymous Variant in. J Cancer (2020) 11(10):2945-56. doi: 10.7150/jca.43010

51. Qian J, Nie W, Lu J, Zhang L, Zhang Y, Zhang B, et al. Racial Differences in Characteristics and Prognoses Between Asian and White Patients With Nonsmall Cell Lung Cancer Receiving Atezolizumab: An Ancillary Analysis of the POPLAR and OAK Studies. Int J Cancer (2020) 146(11):3124-33. doi: $10.1002 /$ ijc. 32717

52. Gu W, Wang N, Qiu Y, Zhang H, Liang J, Zhou T, et al. Molecular Gene Mutation Profiles, TMB and the Impact of Prognosis in Caucasians and East Asian Patients With Lung Adenocarcinoma. Transl Lung Cancer Res (2020) 9(3):629-38. doi: 10.21037/tlcr-20-457

53. Riviere P, Goodman AM, Okamura R, Barkauskas DA, Whitchurch TJ, Lee $\mathrm{S}$, et al. High Tumor Mutational Burden Correlates With Longer Survival in Immunotherapy-Naïve Patients With Diverse Cancers. Mol Cancer Ther (2020) 19(10):2139-45. doi: 10.1158/1535-7163.MCT-20-0161

54. Su J, Yu W, Liu J, Zheng J, Huang S, Wang Y, et al. Fluorescence in Situ Hybridisation as an Ancillary Tool in the Diagnosis of Acral Melanoma: A Review of 44 Cases. Pathology (2017) 49(7):740-9. doi: 10.1016/ j.pathol.2017.08.006

55. Horn S, Figl A, Rachakonda PS, Fischer C, Sucker A, Gast A, et al. TERT Promoter Mutations in Familial and Sporadic Melanoma. Science (2013) 339 (6122):959-61. doi: 10.1126/science.1230062

56. Huang FW, Hodis E, Xu MJ, Kryukov GV, Chin L, Garraway LA. Highly Recurrent TERT Promoter Mutations in Human Melanoma. Science (2013) 339(6122):957-9. doi: 10.1126/science.1229259

57. Bai X, Kong Y, Chi Z, Sheng X, Cui C, Wang X, et al. MAPK Pathway and TERT Promoter Gene Mutation Pattern and Its Prognostic Value in Melanoma Patients: A Retrospective Study of 2,793 Cases. Clin Cancer Res (2017) 23(20):6120-7. doi: 10.1158/1078-0432.CCR-17-0980

58. Luo Y, Zhang Z, Liu J, Li L, Xu X, Yao X, et al. Characterizations of Gene Alterations in Melanoma Patients From Chinese Population. BioMed Res Int (2020) 2020:6096814. doi: 10.1155/2020/6096814

59. Byeon S, Cho HJ, Jang KT, Kwon M, Lee J, Kim ST. Molecular Profiling of Asian Patients With Advanced Melanoma Receiving Check-Point Inhibitor Treatment. ESMO Open (2021) 6(1):100002. doi: 10.1016/ j.esmoop.2020.100002

60. Patel SP, Kurzrock R. PD-L1 Expression as a Predictive Biomarker in Cancer Immunotherapy. Mol Cancer Ther (2015) 14(4):847-56. doi: 10.1158/15357163.MCT-14-0983

61. Munhoz RR, Postow MA. Clinical Development of PD-1 in Advanced Melanoma. Cancer J (2018) 24(1):7-14. doi: 10.1097/PPO.0000000000000299

62. Kaunitz GJ, Cottrell TR, Lilo M, Muthappan V, Esandrio J, Berry S, et al. Melanoma Subtypes Demonstrate Distinct PD-L1 Expression Profiles. Lab Invest (2017) 97(9):1063-71. doi: 10.1038/labinvest.2017.64

63. Castaneda CA, Torres-Cabala C, Castillo M, Villegas V, Casavilca S, Cano L, et al. Tumor Infiltrating Lymphocytes in Acral Lentiginous Melanoma: A
Study of a Large Cohort of Cases From Latin America. Clin Transl Oncol (2017) 19(12):1478-88. doi: 10.1007/s12094-017-1685-3

64. Castaneda CA, Castillo M, Torres-Cabala C, Bernabe LA, Casavilca S, Villegas V, et al. Relationship Between Tumor-Associated Immune Infiltrate and p16 Staining Over Clinicopathological Features in Acral Lentiginous Melanoma. Clin Transl Oncol (2019) 21(9):1127-34. doi: 10.1007/s12094-019-02033-x

65. Edwards J, Wilmott JS, Madore J, Gide TN, Quek C, Tasker A, et al. CD103. Clin Cancer Res (2018) 24(13):3036-45. doi: 10.1158/1078-0432.CCR-172257

66. Edwards J, Ferguson PM, Lo SN, Pires da Silva I, Colebatch AJ, Lee H, et al. Tumor Mutation Burden and Structural Chromosomal Aberrations are Not Associated With T-Cell Density or Patient Survival in Acral, Mucosal, and Cutaneous Melanomas. Cancer Immunol Res (2020) 8(11):1346-53. doi 10.1158/2326-6066.CIR-19-0835

67. Tang B, Chi Z, Chen Y, Liu X, Wu D, Chen J, et al. Safety, Efficacy, and Biomarker Analysis of Toripalimab in Previously Treated Advanced Melanoma: Results of the POLARIS-01 Multicenter Phase II Trial. Clin Cancer Res (2020) 26(16):4250-9. doi: 10.1158/1078-0432.CCR-19-3922

68. Liu D, Schilling B, Sucker A, Livingstone E, Jerby-Arnon L, Zimmer L, et al. Integrative Molecular and Clinical Modeling of Clinical Outcomes to PD1 Blockade in Patients With Metastatic Melanoma. Nat Med (2019) 25 (12):1916-27. doi: 10.1038/s41591-019-0654-5

69. Lee J, Lee SJ, Kim K, Kim ST, Jang KT. Comprehensive Molecular and Clinical Characterization of Asian Melanoma Patients Treated With antiPD-1 Antibody. BMC Cancer (2019) 19(1):805. doi: 10.1186/s12885-019. 6030-5

70. Mao L, Si L, Chi Z, Cui C, Sheng X, Li S, et al. A Randomised Phase II Trial of 1 Month Versus 1 Year of Adjuvant High-Dose Interferon $\alpha$-2b in HighRisk Acral Melanoma Patients. Eur J Cancer (2011) 47(10):1498-503. doi: 10.1016/j.ejca.2011.03.019

71. Wheatley K, Ives N, Hancock B, Gore M, Eggermont A, Suciu S. Does Adjuvant Interferon-Alpha for High-Risk Melanoma Provide a Worthwhile Benefit? A Meta-Analysis of the Randomised Trials. Cancer Treat Rev (2003) 29(4):241-52. doi: 10.1016/S0305-7372(03)00074-4

72. Hauschild A, Grob JJ, Demidov LV, Jouary T, Gutzmer R, Millward M, et al. Dabrafenib in BRAF-Mutated Metastatic Melanoma: A Multicentre, OpenLabel, Phase 3 Randomised Controlled Trial. Lancet (2012) 380(9839):35865. doi: 10.1016/S0140-6736(12)60868-X

73. Ascierto PA, Minor D, Ribas A, Lebbe C, O'Hagan A, Arya N, et al. Phase II Trial (BREAK-2) of the BRAF Inhibitor Dabrafenib (GSK2118436) in Patients With Metastatic Melanoma. J Clin Oncol (2013) 31(26):3205-11. doi: 10.1200/JCO.2013.49.8691

74. Sosman JA, Kim KB, Schuchter L, Gonzalez R, Pavlick AC, Weber JS, et al Survival in BRAF V600-Mutant Advanced Melanoma Treated With Vemurafenib. N Engl J Med (2012) 366(8):707-14. doi: 10.1056/ NEJMoa1112302

75. Wu X, Yu J, Yan J, Dai J, Si L, Chi Z, et al. Pi3k/Akt/mTOR Pathway Inhibitors Inhibit the Growth of Melanoma Cells With Mtor H2189Y Mutations In Vitro. Cancer Biol Ther (2018) 19(7):584-9. doi: 10.1080/ 15384047.2018.1435221

76. Lee B, McArthur GA. CDK4 Inhibitors an Emerging Strategy for the Treatment of Melanoma. Melanoma Manag (2015) 2(3):255-66. doi 10.2217/mmt.15.14

77. Sanz G, Singh M, Peuget S, Selivanova G. Inhibition of p53 Inhibitors: Progress, Challenges and Perspectives. J Mol Cell Biol (2019) 11(7):586-99. doi: $10.1093 / j m c b / m j z 075$

78. Leonardi GC, Candido S, Falzone L, Spandidos DA, Libra M. Cutaneous Melanoma and the Immunotherapy Revolution (Review). Int J Oncol (2020) 57(3):609-18. doi: 10.3892/ijo.2020.5088

79. Ribas A, Puzanov I, Dummer R, Schadendorf D, Hamid O, Robert C, et al. Pembrolizumab Versus Investigator-Choice Chemotherapy for IpilimumabRefractory Melanoma (KEYNOTE-002): A Randomised, Controlled, Phase 2 Trial. Lancet Oncol (2015) 16(8):908-18. doi: 10.1016/S1470-2045(15) 00083-2

80. Hamid O, Robert C, Daud A, Hodi FS, Hwu WJ, Kefford R, et al. Safety and Tumor Responses With Lambrolizumab (anti-PD-1) in Melanoma. N Engl J Med (2013) 369(2):134-44. doi: 10.1056/NEJMoa1305133 
81. Topalian SL, Sznol M, McDermott DF, Kluger HM, Carvajal RD, Sharfman WH, et al. Survival, Durable Tumor Remission, and Long-Term Safety in Patients With Advanced Melanoma Receiving Nivolumab. J Clin Oncol (2014) 32(10):1020-30. doi: 10.1200/JCO.2013.53.0105

82. Schadendorf D, Ascierto PA, Haanen J, Espinosa E, Demidov L, Garbe C, et al. Safety and Efficacy of Nivolumab in Challenging Subgroups With Advanced Melanoma Who Progressed on or After Ipilimumab Treatment: A Single-Arm, Open-Label, Phase II Study (CheckMate 172). Eur J Cancer (2019) 121:144-53. doi: 10.1016/j.ejca.2019.08.014

83. Shoushtari AN, Munhoz RR, Kuk D, Ott PA, Johnson DB, Tsai KK, et al. The Efficacy of Anti-PD-1 Agents in Acral and Mucosal Melanoma. Cancer (2016) 122(21):3354-62. doi: 10.1002/cncr.30259

84. Klemen ND, Wang M, Rubinstein JC, Olino K, Clune J, Ariyan S, et al. Survival After Checkpoint Inhibitors for Metastatic Acral, Mucosal and Uveal Melanoma. J Immunother Cancer (2020) 8(1). doi: 10.1136/jitc-2019-000341

85. Lu M, Wu KH, Trudeau S, Jiang M, Zhao J, Fan E. A Genomic Signature for Accurate Classification and Prediction of Clinical Outcomes in Cancer Patients Treated With Immune Checkpoint Blockade Immunotherapy. Sci Rep (2020) 10(1):20575. doi: 10.1038/s41598-020-77653-3

86. Yarchoan M, Hopkins A, Jaffee EM. Tumor Mutational Burden and Response Rate to PD-1 Inhibition. N Engl J Med (2017) 377(25):2500-1. doi: 10.1056/NEJMc1713444

87. Axelrod ML, Johnson DB, Balko JM. Emerging Biomarkers for Cancer Immunotherapy in Melanoma. Semin Cancer Biol (2018) 52(Pt 2):207-15. doi: 10.1016/j.semcancer.2017.09.004

88. Lawrence MS, Stojanov P, Polak P, Kryukov GV, Cibulskis K, Sivachenko A, et al. Mutational Heterogeneity in Cancer and the Search for New CancerAssociated Genes. Nature (2013) 499(7457):214-8. doi: 10.1038/ nature 12213

89. Nakamura Y, Namikawa K, Yoshino K, Yoshikawa S, Uchi H, Goto K, et al. Anti-PD1 Checkpoint Inhibitor Therapy in Acral Melanoma: A Multicenter Study of 193 Japanese Patients. Ann Oncol (2020) 31(9):1198-206. doi: 10.1016/j.annonc.2020.05.031

90. Si L, Zhang X, Shu Y, Pan H, Wu D, Liu J, et al. A Phase Ib Study of Pembrolizumab as Second-Line Therapy for Chinese Patients With Advanced or Metastatic Melanoma (Keynote-151). Transl Oncol (2019) 12 (6):828-35. doi: 10.1016/j.tranon.2019.02.007

91. Larkin J, Chiarion-Sileni V, Gonzalez R, Grob JJ, Rutkowski P, Lao CD, et al. Five-Year Survival With Combined Nivolumab and Ipilimumab in Advanced Melanoma. N Engl J Med (2019) 381(16):1535-46. doi: 10.1056/ NEJMoa1910836

92. Robert C, Ribas A, Schachter J, Arance A, Grob JJ, Mortier L, et al. Pembrolizumab Versus Ipilimumab in Advanced Melanoma (KEYNOTE006): Post-Hoc 5-Year Results From an Open-Label, Multicentre, Randomised, Controlled, Phase 3 Study. Lancet Oncol (2019) 20(9):123951. doi: 10.1016/S1470-2045(19)30388-2

93. Yu J, Yan J, Guo Q, Chi Z, Tang B, Zheng B, et al. Genetic Aberrations in the CDK4 Pathway Are Associated With Innate Resistance to PD-1 Blockade in Chinese Patients With Non-Cutaneous Melanoma. Clin Cancer Res (2019) 25(21):6511-23. doi: 10.1158/1078-0432.CCR-19-0475

94. Hilke FJ, Sinnberg T, Gschwind A, Niessner H, Demidov G, Amaral T, et al. Distinct Mutation Patterns Reveal Melanoma Subtypes and Influence Immunotherapy Response in Advanced Melanoma Patients. Cancers (Basel) (2020) 12(9). doi: 10.3390/cancers12092359

95. Lerner BA, Stewart LA, Horowitz DP, Carvajal RD. Mucosal Melanoma: New Insights and Therapeutic Options for a Unique and Aggressive Disease. Oncol (Williston Park) (2017) 31(11):e23-32.

96. Mihajlovic M, Vlajkovic S, Jovanovic P, Stefanovic V. Primary Mucosal Melanomas: A Comprehensive Review. Int J Clin Exp Pathol (2012) 5 (8):739-53.

97. Akiyama M, Matsuda Y, Arai T, Saeki H. Clinicopathological Characteristics of Malignant Melanomas of the Skin and Gastrointestinal Tract. Oncol Lett (2018) 16(2):2675-81. doi: 10.3892/ol.2018.8913

98. Aiempanakit K, Chiratikarnwong K, Auepemkiate S, Sriplung H. Clinicopathologic Characteristics and Survival Outcomes of Primary Mucosal Melanomas: A 10-Year Retrospective Analysis From a Single Tertiary Medical Center in Thailand. Dermatologica Sin (2018) 36(3):1402. doi: 10.1016/j.dsi.2017.12.004
99. Lian B, Cui CL, Zhou L, Song X, Zhang XS, Wu D, et al. The Natural History and Patterns of Metastases From Mucosal Melanoma: An Analysis of 706 Prospectively-Followed Patients. Ann Oncol (2017) 28(4):868-73. doi: 10.1093/annonc/mdw694

100. Cui C, Lian B, Zhou L, Song X, Zhang X, Wu D, et al. Multifactorial Analysis of Prognostic Factors and Survival Rates Among 706 Mucosal Melanoma Patients. Ann Surg Oncol (2018) 25(8):2184-92. doi: 10.1245/s10434-018-6503-9

101. Altieri L, Wong MK, Peng DH, Cockburn M. Mucosal Melanomas in the Racially Diverse Population of California. J Am Acad Dermatol (2017) 76 (2):250-7. doi: 10.1016/j.jaad.2016.08.007

102. Nassar KW, Tan AC. The Mutational Landscape of Mucosal Melanoma. Semin Cancer Biol (2020) 61:139-48. doi: 10.1016/j.semcancer.2019.09.013

103. Zou Z, Ou Q, Ren Y, Lv Q, Qin L, Zhao L, et al. Distinct Genomic Traits of Acral and Mucosal Melanomas Revealed by Targeted Mutational Profiling. Pigment Cell Melanoma Res (2020) 33(4):601-11. doi: 10.1111/pcmr.12865

104. Si L, Wang X, Guo J. Genotyping of Mucosal Melanoma. Chin Clin Oncol (2014) 3(3):34. doi: 10.3978/j.issn.2304-3865.2014.07.03

105. Newell F, Kong Y, Wilmott JS, Johansson PA, Ferguson PM, Cui C, et al. Whole-Genome Landscape of Mucosal Melanoma Reveals Diverse Drivers and Therapeutic Targets. Nat Commun (2019) 10(1):3163. doi: 10.1038/ s41467-019-11107-x

106. Ganesh S, Shui S, Craig K, Wang W, Brown BD, Abrams M. Effect of RNAibased $\beta$-Catenin Inhibition on Immunosuppressive Wnt-Activated Tumors in Combination With IDOi/PD-1 Immunotherapy. J Clin Oncol (2018) 36 (15_suppl):e15038-e. doi: 10.1200/JCO.2018.36.15_suppl.e15038

107. DeVito NC, Xiao C, Zhao F, Evans KS, Theivanthiran B, Lewicki J, et al. Paracrine Wnt- $\beta$-Catenin Signaling Inhibition as a Strategy to Enhance the Efficacy of anti-PD-1 Antibody (Ab) Therapy in a Transgenic Model of Melanoma. J Clin Oncol (2017) 35(15_suppl):3053-. doi: 10.1200/ JCO.2017.35.15_suppl.3053

108. Sheng X, Kong Y, Li Y, Zhang Q, Si L, Cui C, et al. GNAQ and GNA11 Mutations Occur in $9.5 \%$ of Mucosal Melanoma and are Associated With Poor Prognosis. Eur J Cancer (2016) 65:156-63. doi: 10.1016/j.ejca.2016.06.019

109. Xu L, Cheng Z, Cui C, Wu X, Yu H, Guo J, et al. Frequent Genetic Aberrations in the Cell Cycle Related Genes in Mucosal Melanoma Indicate the Potential for Targeted Therapy. J Transl Med (2019) 17 (1):245. doi: 10.1186/s12967-019-1987-z

110. Ma M, Dai J, Tang H, Xu T, Yu S, Si L, et al. MicroRNA-23a-3p Inhibits Mucosal Melanoma Growth and Progression Through Targeting Adenylate Cyclase 1 and Attenuating cAMP and MAPK Pathways. Theranostics (2019) 9(4):945-60. doi: 10.7150/thno.30516

111. Adashek J, Szeto C, Sanborn JZ, Reddy S, Toor A, Danielides S, et al. Targetable Immune Checkpoint Molecules may be Significantly Differentially Expressed in Minority Ethnicities. J Clin Oncol (2020) 38:3576-. doi: 10.1200/JCO.2020.38.15_suppl.3576

112. Carvajal RD, Spencer SA, Lydiatt W. Mucosal Melanoma: A Clinically and Biologically Unique Disease Entity. J Natl Compr Canc Netw (2012) 10 (3):345-56. doi: 10.6004/jnccn.2012.0034

113. Yi JH, Yi SY, Lee HR, Lee SI, Lim DH, Kim JH, et al. Dacarbazine-Based Chemotherapy as First-Line Treatment in Noncutaneous Metastatic Melanoma: Multicenter, Retrospective Analysis in Asia. Melanoma Res (2011) 21(3):223-7. doi: 10.1097/CMR.0b013e3283457743

114. Lian B, Si L, Cui C, Chi Z, Sheng X, Mao L, et al. Phase II Randomized Trial Comparing High-Dose IFN- $\alpha 2 b$ With Temozolomide Plus Cisplatin as Systemic Adjuvant Therapy for Resected Mucosal Melanoma. Clin Cancer Res (2013) 19(16):4488-98. doi: 10.1158/1078-0432.CCR-13-0739

115. Yan X, Sheng X, Chi Z, Si L, Cui C, Kong Y, et al. Randomized Phase II Study of Bevacizumab in Combination With Carboplatin Plus Paclitaxel in Patients With Previously Untreated Advanced Mucosal Melanoma. J Clin Oncol (2021) 39:JCO2000902. doi: 10.1200/JCO.20.00902

116. Wong CW, Fan YS, Chan TL, Chan ASW, Ho LC, Ma TKF, et al. BRAF and NRAS Mutations are Uncommon in Melanomas Arising in Diverse Internal Organs. J Clin Pathol (2005) 58(6):640. doi: 10.1136/jcp.2004.022509

117. Dumaz N, Jouenne F, Delyon J, Mourah S, Bensussan A, Lebbé C. Atypical. Cancers (Basel) (2019) 11(8). doi: 10.3390/cancers11081133

118. Tyrrell H, Payne M. Combatting Mucosal Melanoma: Recent Advances and Future Perspectives. Melanoma Manag (2018) 5(3):MMT11. doi: 10.2217/ mmt-2018-0003 
119. Gutiérrez-Castañeda LD, Nova JA, Tovar-Parra JD. Frequency of Mutations in BRAF, NRAS, and KIT in Different Populations and Histological Subtypes of Melanoma: A Systemic Review. Melanoma Res (2020) 30(1):62-70. doi: 10.1097/CMR.0000000000000628

120. Kim KB, Alrwas A. Treatment of KIT-mutated Metastatic Mucosal Melanoma. Chin Clin Oncol (2014) 3(3):35. doi: 10.3978/j.issn.23043865.2014.08.02

121. Hodi FS, Corless CL, Giobbie-Hurder A, Fletcher JA, Zhu M, MarinoEnriquez A, et al. Imatinib for Melanomas Harboring Mutationally Activated or Amplified KIT Arising on Mucosal, Acral, and Chronically Sun-Damaged Skin. J Clin Oncol (2013) 31(26):3182-90. doi: 10.1200/ JCO.2012.47.7836

122. Carvajal RD, Lawrence DP, Weber JS, Gajewski TF, Gonzalez R, Lutzky J, et al. Phase II Study of Nilotinib in Melanoma Harboring KIT Alterations Following Progression to Prior Kit Inhibition. Clin Cancer Res (2015) 21 (10):2289-96. doi: 10.1158/1078-0432.CCR-14-1630

123. Studentova H, Kalabova H, Koranda P, Chytilova K, Kucerova L, Melichar B, et al. Immunotherapy in Mucosal Melanoma: A Case Report and Review of the Literature. Oncotarget (2018) 9(25):17971-7. doi: 10.18632/ oncotarget. 24727

124. D’Angelo SP, Larkin J, Sosman JA, Lebbé C, Brady B, Neyns B, et al. Efficacy and Safety of Nivolumab Alone or in Combination With Ipilimumab in Patients With Mucosal Melanoma: A Pooled Analysis. J Clin Oncol (2017) 35 (2):226-35. doi: 10.1200/JCO.2016.67.9258

125. Hamid O, Robert C, Ribas A, Hodi FS, Walpole E, Daud A, et al. Antitumour Activity of Pembrolizumab in Advanced Mucosal Melanoma: A Post-Hoc Analysis of KEYNOTE-001, 002, 006. Br J Cancer (2018) 119(6):670-4. doi: 10.1038/s41416-018-0207-6

126. Shoushtari AN, Wagstaff J, Ascierto PA, Butler MO, Lao CD, MarquezRodas I, et al. CheckMate 067: Long-Term Outcomes in Patients With Mucosal Melanoma. J Clin Oncol (2020) 38(15_suppl):10019-. doi: 10.1200/ JCO.2020.38.15_suppl.10019

127. Zhang Y, Fu X, Qi Y, Gao Q. A Study of the Clinical Characteristics and Prognosis of Advanced Mucosal and Cutaneous Melanoma in a Chinese Population. Immunotherapy (2019) 11(2):91-9. doi: 10.2217/imt-20180030

128. Zimmer L, Eigentler TK, Kiecker F, Simon J, Utikal J, Mohr P, et al. OpenLabel, Multicenter, Single-Arm Phase II DeCOG-study of Ipilimumab in Pretreated Patients With Different Subtypes of Metastatic Melanoma. J Transl Med (2015) 13:351. doi: 10.1186/s12967-015-0716-5

129. Schaefer T, Satzger I, Gutzmer R. Clinics, Prognosis and New Therapeutic Options in Patients With Mucosal Melanoma: A Retrospective Analysis of 75 Patients. Med (Baltimore) (2017) 96(1):e5753. doi: 10.1097/MD. 0000000000005753

130. Mignard C, Deschamps Huvier A, Gillibert A, Duval Modeste AB, Dutriaux C, Khammari A, et al. Efficacy of Immunotherapy in Patients With Metastatic Mucosal or Uveal Melanoma. J Oncol (2018) 2018:1908065. doi: 10.1093/annonc/mdy289.026

131. Sakaizawa K, Ashida A, Uchiyama A, Ito T, Fujisawa Y, Ogata D, et al. Clinical Characteristics Associated With BRAF, NRAS and KIT Mutations in Japanese Melanoma Patients. J Dermatol Sci (2015) 80(1):33-7. doi: 10.1016/ j.jdermsci.2015.07.012

132. Tzen C-Y, Wu Y-H, Tzen C-Y. Characterization of KIT Mutation in Melanoma. Dermatologica Sin (2014) 32(1):7-12. doi: 10.1016/j.dsi. 2013.05.005
133. Simonetti O, Lucarini G, Rubini C, Lazzarini R, DI Primio R, Offidani A. Clinical and Prognostic Significance of Survivin, AKT and VEGF in Primary Mucosal Oral Melanoma. Anticancer Res (2015) 35(4):2113-20.

134. Cui C, Tang B, Guo J. Chemotherapy, Biochemotherapy and anti-VEGF Therapy in Metastatic Mucosal Melanoma. Chin Clin Oncol (2014) 3(3):36. doi: 10.3978/j.issn.2304-3865.2014.07.02

135. Kim KB, Sosman JA, Fruehauf JP, Linette GP, Markovic SN, McDermott DF, et al. BEAM: A Randomized Phase II Study Evaluating the Activity of Bevacizumab in Combination With Carboplatin Plus Paclitaxel in Patients With Previously Untreated Advanced Melanoma. J Clin Oncol (2012) 30 (1):34-41. doi: 10.1200/JCO.2011.34.6270

136. Yasuda S, Sho M, Yamato I, Yoshiji H, Wakatsuki K, Nishiwada S, et al. Simultaneous Blockade of Programmed Death 1 and Vascular Endothelial Growth Factor Receptor 2 (VEGFR2) Induces Synergistic Anti-Tumour Effect In Vivo. Clin Exp Immunol (2013) 172(3):500-6. doi: 10.1111/cei.12069

137. Guo J, Sheng X, Si L, Kong Y, Chi Z, Cui C, et al. A Phase Ib Study of JS001, a Humanized IgG4 mAb Against Programmed Death-1 (PD-1) Combination With Axitinib in Patients With Metastatic Mucosal Melanoma. J Clin Oncol (2018) 36(15_suppl):9528-. doi: 10.1200/JCO.2018.36.15_suppl.9528

138. Sheng X, Yan X, Chi Z, Si L, Cui C, Tang B, et al. Axitinib in Combination With Toripalimab, a Humanized Immunoglobulin G. J Clin Oncol (2019) 37 (32):2987-99. doi: 10.1200/JCO.19.00210

139. Taylor MH, Vogelzang NJ, Cohn AL, Stepan DE, Shumaker RC, Dutcus CE, et al. Phase Ib/II Trial of Lenvatinib Plus Pembrolizumab in Advanced Melanoma. J Clin Oncol (2019) 37(8_suppl):15-. doi: 10.1200/JCO.2019.37.8_suppl.15

140. Si L, Sheng X, Mao L, Li C, Wang X, Bai X, et al. A Phase II Study of Vorolanib (CM082) in Combination With Toripalimab (JS001) in Patients With Advanced Mucosal Melanoma. J Clin Oncol (2020) 38 (15_suppl):10040-. doi: 10.1200/JCO.2020.38.15_suppl.10040

141. Li J, Kan H, Zhao L, Sun Z, Bai C. Immune Checkpoint Inhibitors in Advanced or Metastatic Mucosal Melanoma: A Systematic Review. Ther Adv Med Oncol (2020) 12:1758835920922028. doi: 10.1177/1758835920922028

142. Namikawa K, Kiyohara Y, Takenouchi T, Uhara H, Uchi H, Yoshikawa S, et al. Efficacy and Safety of Nivolumab in Combination With Ipilimumab in Japanese Patients With Advanced Melanoma: An Open-Label, Single-Arm, Multicentre Phase II Study. Eur J Cancer (2018) 105:114-26. doi: 10.1016/ j.ejca.2018.09.025

Conflict of Interest: JG is a member of the advisory board/consultant of MSD, Roche, Pfizer, Bayer, Novartis, Simcere, Shanghai Junshi Bioscience, Oriengene. $\mathrm{LZ}$ is an employee of MSD China.

The authors declare that this review received funding from MSD China. The funder had the following involvement in the review: formally reviewing a penultimate draft.

The remaining authors declare that the research was conducted in the absence of any commercial or financial relationships that could be construed as a potential conflict of interest.

Copyright (c) 2021 Mao, Qi, Zhang, Guo and Si. This is an open-access article distributed under the terms of the Creative Commons Attribution License (CC BY). The use, distribution or reproduction in other forums is permitted, provided the original author(s) and the copyright owner(s) are credited and that the original publication in this journal is cited, in accordance with accepted academic practice. No use, distribution or reproduction is permitted which does not comply with these terms. 